\title{
On Thermodynamics and Intrinsically Equilibrated Materials $\left(^{*}\right)(* *)$.
}

\author{
Bernard D. Coleman - Davm R. Owen (Pittsburgh, Pa. U.S.A.)
}

Dedicated to DaRIo Graffi on his 70th Birthday

\begin{abstract}
Summary. - The existence and uniqueness of free energy functions is demonstrated for a class of materials broad enough to contain as special cases those of the theory of finite elasticity, the theory of hypo-elasticity, and the theory of internal state vasiables for which the path of evalution is invariant under rescalings of time.
\end{abstract}

\section{1. - Introduction.}

We have recently proposed and explored a mathematical theory of thermodynamics in which the existence of certain calorie functions of state, such as entropy and free energy, is deduced from "cyclic " inequalities for a class of generalized integrals called "actions " [1], [2]. Here, after summarizing the basic definitions and listing some of the main theorems of our mathematical theory, we apply the theory to a class of systems called "intrinsically equilibrated elements". This class of systems, whieh is closely related to a class studied by OWEN and WILLIAMS [3] from another point of view, is broad enough to contain as special eases material elements obeying the constitutive equations of the following theories from continuum mechanics: the classical theory of isothermal finite elastic deformations, NoLI's recently proposed generalization [4] of TrUeSDELL's theory of hypo-elasticity [5], and certain theories of materials with internal state variables whose path of evolution is invariant under temporal rescalings. We show that if an intrinsically equilibrated element is compatible with thermodynamics, then it has a Helmholtz free energy function $\psi$ which is unique (to within an additive constant), and $\psi$ enters into a relation which generalizes the classical "stress relation " of elasticity theory. For simplicity of exposition, we confine the present discussion to isothermal cases, i.e. to cases in which the temperature gradient vanishes and the temperature remains constant in time at the material element under consideration.

(*) Entrata in Redazione il 24 giugno 1975.

(**) This research was supported by the U. S. National Science Foundation under Grant GP-32951 X2.

13 - Annali di Motematica 


\section{2. - On the general theory of systems and actions.}

Let $(\Sigma, \Pi)$ be an ordered pair in which $\Sigma$ is a topological space and $\Pi$ a set of objects such that each $P$ in $I$ determines a continuous mapping $\varrho_{P}$ of a non-empty open subset $\mathcal{D}(P)$ of $\Sigma$ onto a subset $R(P)$ of $\Sigma$. If $(I)$ for each $\sigma$ in $\Sigma$, the set

$$
\Pi_{\sigma} \stackrel{\underline{A}}{=}\left\{\varrho_{P} \sigma \mid P \in \Pi, \sigma \in \mathfrak{D}(P)\right\}
$$

is dense in $\Sigma$, and $(\boldsymbol{I I})$ on the set

$$
\mathfrak{T}=\left\{\left(P^{\prime \prime}, P^{\prime}\right) \in \Pi \times \Pi \mid \mathfrak{D}\left(P^{\prime \prime}\right) \cap \Re\left(P^{\prime}\right) \neq \emptyset\right\}
$$

there is defined a $\Pi$-valued function $\left(P^{\prime \prime}, P^{\prime}\right) \mapsto P^{\prime \prime} P^{\prime}$ such that

$$
\mathfrak{D}\left(P^{\prime \prime} P^{\prime}\right)=\varrho_{P^{\prime}}^{-1}\left(\mathfrak{D}\left(P^{\prime \prime}\right) \cap \mathfrak{R}\left(P^{\prime}\right)\right),
$$

and, for each $\sigma$ in $\mathfrak{D}\left(P^{\prime \prime} P^{\prime}\right)$,

$$
\varrho_{P^{\prime} P^{\prime \prime}} \sigma=\varrho_{P^{\prime}} \varrho_{P^{\prime \prime}} \sigma
$$

then $(\Sigma, \Pi)$ is called a system, the elements $\sigma$ of $\Sigma$ are called states, and the elements $P$ of $I I$ are called processes; for a process $P$, the function $\varrho_{P}$ is referred to as the transformation induced by $P$; for a state $\sigma, \Pi \sigma$ is called the set of states accessible from $\sigma$; each element $\left(P^{\prime \prime}, P^{\prime}\right)$ of $\mathfrak{T}$ is called an ordered pair of successively applicable processes, and the value $P^{\prime \prime} P^{\prime}$ of the function $\left(P^{\prime \prime}, P^{\prime}\right) \mapsto P^{\prime \prime} P^{\prime}$ of $(2.3)$ and $(2.4)$ is referred to as the process resulting from the successive application of $P^{\prime}$ and $P^{\prime \prime}$; for the set of all ordered pairs $(P, \sigma)$ with $P$ in $\Pi$ and $\sigma$ in $\mathscr{D}(P)$, one writes $\Pi \diamond \Sigma$, i.e.,

$$
\Pi \diamond \Sigma \stackrel{\Delta}{=}\{(P, \sigma) \in \Pi \times \Sigma \mid \sigma \in \mathscr{D}(P)\} .
$$

An action $\mathfrak{a}$ for a system $(\Sigma, \Pi)$ is a function from $\Pi \diamond \Sigma$ into $\mathbb{R}$ obeying the following two conditions:

Additivity: if $\left(P^{\prime \prime}, P^{\prime}\right)$ is in $\mathfrak{S} \subset \Pi \times \Pi$ and $\sigma$ is in $\mathcal{D}\left(P^{\prime \prime} P^{\prime}\right)$, then

$$
\mathfrak{a}\left(P^{\prime \prime} P^{\prime}, \sigma\right)=\mathfrak{a}\left(P^{\prime}, \sigma\right)+\mathfrak{a}\left(P^{\prime \prime}, \varrho_{P^{\prime}} \sigma\right) ;
$$

Continuity: for each $P$ in $\Pi$, the function $\mathfrak{a}(P, \cdot): \mathscr{D}(P) \rightarrow \mathbb{R}$ is continuous. When $\mathfrak{a}$ is an action we refer to $\mathfrak{a}(P, \sigma)$ [with, of course, $(P, \sigma)$ in $\Pi \diamond \Sigma$ ] as the supply of $\mathfrak{a}$ on going from $\sigma$ to $Q_{P} \sigma$ via the process $P$.

There are systems for which two processes $P^{\prime}, P^{\prime \prime}$ can induce the same transformation $\varrho_{P^{\prime \prime}}=\varrho_{P^{\prime}}$ but can give different values to an action $a$. Hence, one must bear in mind the distinction between processes and their induced transformations. 
However, because the theory of systems and actions is more concisely described if one does not continually render this distinction explicit, we usually simplify our notation and write $P \sigma$ for $\varrho_{P} \sigma$. For a pair $\left(P^{\prime \prime}, P^{\prime}\right)$ in $\mathfrak{S}$, we often write $P^{\prime \prime} P^{\prime} \sigma$ for $\varrho_{P^{\prime \prime} p^{\prime}} \sigma$, which, by (2.4), is the same as $\varrho_{p^{\prime \prime}} \varrho_{P^{\prime}} \sigma$. In accord with these conventions, $(2.6)$ can be written

$$
\mathfrak{a}\left(P^{\prime \prime} P^{\prime}, \sigma\right)=\mathfrak{a}\left(P^{\prime}, \sigma\right)+\mathfrak{a}\left(P^{\prime \prime}, P^{\prime} \sigma\right) .
$$

When discussing an action $\mathfrak{a}$, if we write the expressions $\mathfrak{a}(P, \sigma)$ or $P \sigma\left(=\varrho_{P} \sigma\right)$, we automatically assume that $\sigma$ is in $\mathfrak{D}(P)$, i.e. that $(P, \sigma)$ is in $\Pi \diamond \Sigma$, and if we write $P^{\prime \prime} P^{\prime}$ we automatically assume that $\mathfrak{D}\left(P^{\prime \prime}\right) \cap \mathfrak{R}\left(P^{\prime}\right)$ is not empty, i.e. that $\left(P^{\prime \prime}, P^{\prime}\right)$ is in $\mathfrak{T}$; for it is only under these circumstances that the expressions $\mathfrak{a}(P, \sigma)$, $P \sigma$, and $P^{\prime \prime} P^{r}$ can have meaning in the present theory.

If $z$ is an action for $(\Sigma, \Pi)$, and $\sigma^{0}$ is in $\Sigma$, we say that $z$ has the dissipation property at $\sigma^{0}$ if and only if for each $\varepsilon>0$ there is a neighborhood $\left(^{1}\right) \mathcal{O}$ of $\sigma^{0}$ such that whenever $P \sigma^{0}$ is in $\mathcal{O}$ there holds

$$
z\left(P, \sigma^{0}\right)>-\varepsilon .
$$

In terms not quite precise, an action $z$ has the dissipation property at a state $\sigma^{0}$ if the supply of $z$ on going from $\sigma^{0}$ to any state in a small neighborhood $\mathcal{O}$ of $\sigma^{0}$ is approximately positive for all processes which effect such a transition. If $z$ has the dissipation property at $\sigma^{0}$, then, for each process $P$ such that $P \sigma^{0}=\sigma^{0}$ (of course, there may not exist even one such process), there holds $z\left(P, \sigma^{0}\right) \geqslant 0$.

A real-valued function $Z$ on a dense subset 3 of $\Sigma$ is called a lower potential for a given action $z$ if whenever $\sigma_{1}$ and $\sigma_{2}$ are in $\tilde{z}$ there is, for each $\varepsilon>0$, a neighborhood $\mathcal{O}$ of $\sigma_{2}$ such that, for every process $P$ with $P \sigma_{1}$ in $\mathcal{O}$, there holds

$$
Z\left(\sigma_{2}\right)-Z\left(\sigma_{1}\right)<z\left(P, \sigma_{1}\right)+\varepsilon .
$$

It is easily seen that if $Z$ is a lower potential for $z$ and if $\sigma$ and $P \sigma$ are in the domain of $Z$, then,

$$
Z(P \sigma)-Z(\sigma) \leqslant z(P, \sigma)
$$

The main general theorems about the dissipation property and lower potentials are stated below; in each of these theorems it is assumed that $z$ is an action for a given system $(\Sigma, \Pi)$.

THeOREM $1\left({ }^{2}\right)$. - If $z$ has the dissipation property at a state $\sigma^{0}$, then $z$ has that property at all states accessible from $\sigma^{0}$.

( $\left.{ }^{1}\right)$ Here, as in [1] and [2], by a "neighborhood " of a state $\sigma^{0}$ we mean an open subset of $\Sigma$ containing $\sigma^{0}$.

(2) [2], Thm. 3.1; see also [1], Remark 3.1. 
Theorem $2\left({ }^{3}\right)$. - If $z$ has a lower potential $Z$, then $z$ has the dissipation properity at every state in the domain of $Z$.

Less trivially, there holds

THEOREM $3\left({ }^{4}\right)$. - If there is a state $\sigma^{0}$ at which $z$ has the dissipation property, then $z$ has a lower semicontinuous lower potential whose domain contains all states accessible from $\sigma^{\circ}$.

\section{3. - Intrinsically equilibrated elements.}

Let $\mathcal{L}$ be the set of all (second-order) tensors on the translation space $\mathcal{V}$ of the usual three-dimensional Euclidean point space, let $\delta$ be the set of symmetric tensors in $\mathfrak{L}$, and let $\mathfrak{B}$ be an open connected subset of a given (not necessarily finitedimensional) Banach space $\mathfrak{U}$ with norm $\|\cdot\|$. We assume that there is given a function $\boldsymbol{K}$ on $\mathfrak{B}$ whose value is, for each $\sigma$ in $\mathfrak{B}$, a linear transformation $K(\sigma)[\cdot]$ of $\mathfrak{L}$ such that the function $(\sigma, L) \mapsto K(\sigma)[L]$ is a locally Lipschitz continuous mapping of $\mathfrak{B} \times \mathfrak{L}$ into $\mathfrak{U}$. We also assume that there is given a (distinguished) element $\sigma^{0}$ in $\mathfrak{B}$, which we shall later refer to as «the standard state ». We say that an $\mathfrak{L}$-valued function $L_{t}$ on $[0, t)$, with $0<t<\infty$, is piecewise continuous if $L_{t}(\tau+) \triangleq \lim _{\zeta \rightarrow \tau+} L_{t}(\zeta)$ exists and equals $L_{t}(\tau)$ for each $\tau$ in $[0, t), L_{t}(\tau-) \triangleq \lim _{\zeta \rightarrow \tau-} L_{t}(\zeta)$ exists for each $\tau$ in $(0, t]$, and $L_{t}(\tau-)=L_{t}(\tau+)$ at all but a finite number of points in $(0, t)$. For each element $\sigma^{*}$ of $\mathfrak{B}$ we write $\Psi\left(\sigma^{*}\right)$ for the set of all piecewise continuous functions $L_{t}:[0, t) \rightarrow \mathfrak{L}$, with $0<t<\infty$, which are such that the differential equation

$$
\dot{\sigma}(\tau)=\boldsymbol{K}(\sigma(\tau))\left[L_{t}(\tau)\right]
$$

has a (classical) solution on $[0, t]$ obeying the initial condition

$$
\sigma(0)=\sigma^{*}
$$

and lying in $\mathfrak{B}$. (Using the Lipsehitz theory of ordinary differential equations, one can easily show that if a solution $\sigma(\cdot):[0, t] \rightarrow \mathfrak{B}$ of $(3.1)$ with $\sigma(0)=\sigma^{*}$ exists, it is unique.)

Now, let $\Sigma$ be the set of all elements $\sigma$ of $\mathfrak{B}$ which occur as values $\sigma(\tau)$ of solutions of (3.1) with $\sigma(0)=\sigma^{0}$ and with $L_{t}$ in $\Psi\left(\sigma^{0}\right)$; let $\Sigma$ have the metric (and hence the topology) induced by the norm $\|\cdot\|$; and put

$$
\Pi \stackrel{\Delta}{=} \bigcup_{\sigma \in \Sigma} \Psi(\sigma)
$$

(3) [3], Thm. 3.2; see also [1], Thm. 3.2.

(4) [2], Thm. 3.3; see also [1], Thm. 3.3. 
For each $L_{t}$ in $\Pi$, let $\mathfrak{D}\left(L_{t}\right)$ be the set of all $\sigma$ in $\Sigma$ for which $L_{t}$ is in $\Psi(\sigma)$, and for each such $\sigma$ let $L_{t} \sigma$ (i.e. $\varrho_{L_{t}} \sigma$ ) be the value at time $t$ of the (unique) solution of (3.1) with $\sigma(0)=\sigma$. Let $R\left(L_{t}\right)$ be the range of $\varrho_{L_{t}}: \mathfrak{D}\left(L_{t}\right) \rightarrow \Sigma$. [It is not diffcult to show that $\mathfrak{D}\left(L_{t}\right)$ is open in $\mathfrak{B}$, and hence open in $\Sigma$, and that $\varrho_{s_{t}}$ is a continuous mapping of $\mathfrak{D}\left(L_{t}\right)$ onto $\left.\mathcal{R}\left(L_{t}\right\rangle^{\prime}\right]$ For each pair $\left(L_{t_{2}}, L_{t_{1}}\right)$ of elements of $\Pi$ for which $\mathfrak{D}\left(L_{t_{2}}\right)$ intersects $\mathcal{R}\left(L_{t_{1}}\right)$, let $L_{t_{2}} L_{t_{1}}$ be the function $L_{t_{1}+t_{2}}:\left[0, t_{1}+t_{2}\right) \rightarrow \mathcal{L}$ defined by

$$
L_{t_{1}+t_{2}}(\tau) \triangleq \begin{cases}L_{t_{1}}(\tau) & \text { for } \tau \in\left[0, t_{1}\right), \\ L_{t_{2}}\left(\tau-t_{1}\right) & \text { for } \tau \in\left[t_{1}, t_{1}+t_{2}\right) .\end{cases}
$$

The following remark is easily verified.

REMARK 1. - A pair $(\Sigma, \Pi)$ constructed from $K$ and $\sigma^{0}$ as described above is a system, in the sense of the general theory of Section 2, with the elements $\sigma$ of $\Sigma$ the states and the elements $L_{t}$ of $I I$ the processes. Such a system is called an intrinsically equilibrated element, $\boldsymbol{K}$ is called the rate function for the element, and $\sigma^{0}$ is called the standard state. For an intrinsically equilibrated element $(\Sigma, \Pi)$, the set of states accessible from an arbitrary state $\sigma$ in $\Sigma$ is equal to all of $\Sigma$, i.e.

$$
\Pi \sigma \stackrel{A}{=}\left\{L_{t} \sigma \mid L_{t} \in \Pi, \sigma \in \mathfrak{D}\left(L_{t}\right)\right\}=\Sigma,
$$

and the process $L_{t_{2}} L_{l_{1}}$ resulting from the successive application of two processes $L_{t_{1}}$ and $L_{t_{2}}$, with $\mathscr{D}\left(L_{t_{2}}\right) \cap \mathcal{R}\left(L_{t_{1}}\right) \neq \emptyset$, is just the function $L_{t_{1}+t_{3}}$ defined in (3.4).

We assume that with each intrinsically equilibrated element there is associated a locally Lipschitz continuous function $T: \Sigma \rightarrow \delta$ called the stress function. The value $T(\sigma)$ of $T$ is interpreted as the Cauchy stress divided by the mass density.

REMARK 2. - A familiar example of an intrinsically equilibrated element is given by Truesdelis's theory of hypo-elastic materials [5], [6]. For an element of such a material each state $\sigma$ is an ordered pair $(F, S)$ with $F$ an invertible tensor, interpreted as the deformation gradient, and $S$ a symmetric tensor (i.e. an element of $\mathcal{S}$ ), interpreted as the Cauchy stress tensor. In that theory $T$ reduces to the function

$$
\sigma=(F, S) \mapsto v_{r}|\operatorname{det} F| S=T(\sigma)
$$

with $v_{r}$ a constant equal to the specific volume in the reference configuration, and the differential equation (3.1) takes the form

$$
\begin{aligned}
& \dot{F}(\tau)=L_{t}(\tau) F(\tau), \\
& \dot{S}(\tau)=H(F(\tau), S(\tau))\left[D_{t}(\tau)\right]+W_{t}(\tau) S(\tau)-S(\tau) W_{t}(\tau),
\end{aligned}
$$


with

$$
\begin{gathered}
2 D_{t}(\tau)=L_{t}(\tau)+L_{t}(\tau)^{T}, \\
2 W_{t}(\tau)=L_{t}(\tau)-L_{t}(\tau)^{T},
\end{gathered}
$$

and with $\boldsymbol{H}$ a given continuously differentiable function whose value is, for each pair $(F, S)$, a linear transformation $H(F, S)[\cdot]$ of $\delta$ into $S$. Bernstein [7] was apparently the first to point out that the set $\Sigma$ of states, $(F, S)$ of a hypo-elastic material depends upon the choice of the standard state $\sigma^{0}=\left(F^{0}, S^{0}\right)\left(^{5}\right)$. Note that for a hypo-elastic element, $\mathfrak{B}$ and hence $\Sigma$ is a subset of the finite dimensional Banach space $\mathcal{L} \oplus \mathcal{S}$. (In TRUESDELL's original presentation of hypo-elasticity [5], it was assumed that $\boldsymbol{H}$ is independent of its first variable $F$, i.e. that $(3.7)_{2}$ can be written

$$
\dot{S}(\tau)=H(S(\tau))\left[D_{i}(\tau)\right]+W_{t}(\tau) S(\tau)-S(\tau) W_{i}(\tau)
$$

the formulation given here is essentially equivalent to that recently proposed by NoLI [4].)

REMARK 3. - A very general type of intrinsically equilibrated element is obtained by assuming that each state $\sigma$ is an ordered pair $(F, \Omega)$, with $F$ again an invertible tensor interpreted as the deformation gradient, but with $\Omega$ now an element of some Banach space $\mathfrak{W}$, not necessarily isomorphic to $S$. Here $T(\sigma)=T(F, \Omega)$, and in place of (3.7) we have, for (3.1),

$$
\begin{aligned}
& \dot{F}(\tau)=L_{t}(\tau) F(\tau) \\
& \dot{\Omega}(\tau)=\mathbf{B}(F(\tau), \Omega(\tau))\left[L_{t}(\tau)\right]
\end{aligned}
$$

with $B(\cdot)[\cdot]$ a given locally Lipschitz continuous function such that, for each pair $(F, \Omega), \mathrm{B}(F, \Omega)[\cdot]$ is a linear mapping of $\mathcal{L}$ into $\mathfrak{W}$. For such a material element, $\mathfrak{B} \subset \mathfrak{L} \oplus \mathfrak{W}$. When $\mathfrak{W}$ is finite-dimensional and $\Omega \neq S$, one may be tempted to call $\Omega$ the vector of internal state variables. For an elastic element, $\sigma=F$, and $\mathbf{B}$ can be taken to be zero.

For each pair $\left(L_{i}, \sigma\right)$ in $\Pi \diamond \Sigma$, i.e. with $L_{t}$ in $\Pi$ and $\sigma$ in $\mathscr{D}\left(L_{t}\right)$, the number

$$
\mathfrak{w}\left(L_{t}, \sigma\right) \triangleq \int_{0}^{t} T(\sigma(\tau)) \cdot L_{t}(\tau) d \tau
$$

is called the work done by the stress on going from $\sigma$ to $L_{t} \sigma$ via the process $L_{t}$. Of course, $\sigma(\tau)$ is here the value at time $\tau$ of the solution of (3.1) with initial state $\sigma$, i.e. with $\sigma(0)=\sigma$. (For the inner product $A \cdot B$ of two elements, $A$ and $B$ of $\mathcal{L}$, we take trace $\left(A B^{T}\right)$ with ${ }^{T}$ the transposition operator.) Since the inner product of a sym-

(5) See also the more recent discussions of Truesdell \& NoLL [6] and NoLL [4]. 
metric tensor (in $\mathfrak{L}$ ) with a skew tensor (in $\mathfrak{L}$ ) is zero, $(3.10)$ can be written

$$
\mathfrak{w}\left(L_{t}, \sigma\right)=\int_{0}^{t} T(\sigma(\tau)) \cdot D_{t}(\tau) d \tau
$$

with $D_{t}(\tau)$, called the "stretching tensor ", equal to the symmetric part of $L_{t}$, i.e. defined as in $(3.8)_{1}$.

It is a straightforward exercise to verify

REMARK 4. - For each intrinsically equilibrated element $(\Sigma, \Pi)$, the function $\mathfrak{w}: \Pi \diamond \Sigma \rightarrow \mathbb{R}$, defined in (3.10), is an action in the sense of Section 2 .

We call w the work action for $(\Sigma, \Pi)$.

To each process $L_{t}$ of an intrinsically equilibrated element there corresponds a process ${ }_{R} L_{t}:[0, t) \rightarrow \mathfrak{L}$ which is called the reversal of $L_{t}$ and is defined as follows:

$$
{ }_{n} L_{t}(\tau)=\lim _{\alpha \rightarrow \tau++} L_{t}(t-\alpha), \quad \tau \in[0, t) .
$$

(Thus, ${ }_{R} L_{t}(\tau)=L_{t}(t-\tau)$, except at the finite number of points at which $L_{t}$ is discontinuous and at those points $\left.{ }_{R} L_{t}(\tau)={ }_{R} L_{t}(\tau+).\right)\left({ }^{6}\right)$ One may easily verify that for every intrinsically equilibrated element $(\Sigma, \Pi)$ there holds

REMARK 5, - If $L_{t}$ is in $\Pi$, then (i) ${ }_{R} L_{t}$ is in $\Pi$, (ii) $\mathfrak{D}\left({ }_{R} L_{t}\right)=\mathfrak{R}\left(L_{t}\right)$, (iii) $\mathfrak{R}\left({ }_{R} L_{t}\right)=$ $=\mathfrak{D}\left(L_{t}{ }^{\prime},(\mathrm{iv}){ }_{R} L_{t} \sigma_{2}=\sigma_{1}\right.$ if and only if $L_{t} \sigma_{1}=\sigma_{2},(\mathrm{v}){ }_{R R} L_{t}=L_{t}$, and (vi)

$$
\mathfrak{w}\left({ }_{R} L_{t}, \sigma_{2}\right)=-\mathfrak{w}\left(L_{t}, \sigma_{1}\right)
$$

whenever $\sigma_{2}=\dot{L}_{t} \sigma_{1}$.

We say that an intrinsically equilibrated element is compatible with thermodynamics if its work action $w$ has the dissipation property at the standard state $\sigma^{0}$.

In view of (3.5) and Theorems 1 and 3 , we make the following observation.

REMARK 6. - If an intrinsically equilibrated element $(\Sigma, \Pi)$ is compatible with thermodynamies, its work action $\mathfrak{w}$ has the dissipation property at every state $\sigma$ in $\Sigma$, and there exists a lower semicontinuous function $\psi: \Sigma \rightarrow \mathbb{R}$ which is a lower potential for tw.

A lower potential for $\mathfrak{w}$ which has all of $\Sigma$ for its domain is called a free energy function (or a "Helmholtz free energy function ").

The following theorem shows that a general intrinsically equilibrated materiai shares with its special prototype, the elastic material, the property of having at most one Helmholtz free energy function $\psi$ which is "normalized "to vanish at a standard state. Moreover, here, as for an elastic material, $\psi$ serves, in a certain sense, as a "potential" for the work.

$\left.{ }^{6}\right)$ A related concept of "reversed process" was employed by NoLL [4]. 
THEoRem 4. - Each intrinsically equilibrated element compatible with thermodynamies has precisely one free energy function $\psi$ which vanishes at the standard state $\sigma^{0}$; this function $\psi$ is lower semicontinuous and obeys the equation

$$
\psi\left(\sigma_{2}\right)-\psi\left(\sigma_{1}\right)=\mathfrak{w}\left(L_{t}, \sigma_{1}\right)
$$

for every pair of states $\sigma_{1}, \sigma_{2}$ and process $L_{t}$ with $L_{i} \sigma_{1}=\sigma_{2}$.

In another notation, (3.14) takes the form

$$
\psi(\sigma(t))-\psi(\sigma(0))=\int_{0}^{t} T(\sigma(\tau)) \cdot L_{t}(\tau) d \tau=\int_{0}^{t} T(\sigma(\tau)) \cdot D_{t}(\tau) d \tau
$$

We refer to the function $\psi$ determined by wo in accord with. Theorem 4 as "the free energy function " of the intrinsically equilibrated element.

Proof of Theorem 4. - By (2.9), every free energy function for the action $\mathfrak{w}$ obeys the relation

$$
\psi\left(\sigma_{2}\right)-\psi\left(\sigma_{1}\right) \leqslant \mathfrak{w}\left(L_{t}, \sigma_{1}\right)
$$

whenever $\sigma_{2}=L_{t} \sigma_{1}$. Let ${ }_{R} L_{t}$ be the reversal of $L_{t}$, and note that, by Remark 5 , whenever $\sigma_{2}=L_{t} \sigma_{1}$ there holds $\sigma_{1}={ }_{R} L_{t} \sigma_{2}$, and hence, by (2.9) and (3.13),

$$
\psi\left(\sigma_{1}\right)-\psi\left(\sigma_{2}\right) \leqslant \mathfrak{w}\left({ }_{R} L_{t}, \sigma_{2}\right)=-\mathfrak{w}\left(L_{t}, \sigma_{1}\right)
$$

i.e.

$$
\psi\left(\sigma_{2}\right)-\psi\left(\sigma_{1}\right) \geqslant \mathfrak{w}\left(L_{t}, \sigma_{1}\right)
$$

The relations (3.16) and (3.18) obviously yield (3.14), and hence every free energy function for an intrinsically equilibrated element $(\Sigma, \Pi)$ obeys (3.14). Since a free energy function remains a free energy function after addition of a constant, Remark 6 tells us that if $(\Sigma, \Pi)$ is compatible with thermodynamies, $(\Sigma, \Pi)$ does have a free energy function with $\psi\left(\sigma^{0}\right)=0$. By (3.14), such a free energy funetion must obey

$$
\psi(\sigma)=\mathfrak{w}\left(L_{t}, \sigma^{0}\right)
$$

whenever $\sigma=L_{t} \sigma^{0}$. However, by (3.5), for every state $\sigma$ in $\Sigma$ there holds $\sigma=L_{t} \sigma^{0}$ for some $L_{t}$ in $\Pi$, and therefore there can be at most one function $\psi$ on $\Sigma$ obeying obeying (3.19). Thus, if $(\Sigma, \Pi)$ is compatible with thermodynamics, $(\Sigma, \Pi)$ has exactly one free energy function $\psi$ with $\psi\left(\sigma^{0}\right)=0$; moreover, by Remark 6 , this function $\psi$ is lower semicontinuous; q.e.d. 
As an immediate corollary to Theorem 4, we have

THEOREM 5. - The work action $\mathfrak{w}$ of an intrinsically equilibrated element $(\Sigma, \Pi)$ which is compatible with thermodynamics, has the following special properties:

(1) it vanishes on every "closed path " in the sense that

$$
\sigma_{1}=L_{t} \sigma_{1} \Rightarrow \mathfrak{w}\left(L_{t}, \sigma_{1}\right)=0
$$

(2) it is "path independent" in the sense that if $L_{t}$ and $M_{t^{\prime}}$ are two processes which have a given state $\sigma_{1}$ in their domains $\mathfrak{D}\left(L_{t}\right)$ and $\mathfrak{D}\left(M_{t^{\prime}}\right)$, then

$$
L_{t} \sigma_{1}=M_{t^{\prime}} \sigma_{1} \Rightarrow \mathfrak{w}\left(L_{t}, \sigma_{1}\right)=\mathfrak{w}\left(M_{t^{\prime}}, \sigma_{1}\right)
$$

Let $L_{t}:[0, t) \rightarrow \mathcal{L}$ be in $\Pi$; for each $\tau$ in $(0, t]$, let $L_{\tau}$ be the restriction of $L_{t}$ to $[0, \tau)$; and note that $L_{\tau}$ is then in $I I$ with $\mathscr{D}\left(L_{\tau}\right) \supset \mathscr{D}\left(L_{t}\right)$. For each state $\sigma$ in $\mathscr{D}\left(L_{t}\right)$ and for each $\tau$ in $(0, t]$, there holds, by $(3.15)$,

$$
\psi\left(L_{\tau} \sigma\right)-\psi(\sigma)=\int_{0}^{\tau} T\left(L_{\zeta} \sigma\right) \cdot L_{t}(\zeta) d \zeta
$$

and this implies that

$$
\delta \psi\left(\sigma ; L_{t}\right) \stackrel{\Delta}{=} \lim _{\tau \rightarrow 0} \frac{1}{\tau}\left[\psi\left(L_{\tau}(\sigma)\right)-\psi(\sigma)\right]
$$

exists and obeys

$$
\delta \psi\left(\sigma ; L_{t}\right)=T(\sigma) \cdot L_{t}(0)
$$

This last equation tells us, moreover, that $\delta \psi\left(\sigma, L_{t}\right)$ depends on the function $L_{t}$ only through its initial value $L_{t}(0)$; i.e. if $L_{t}$ and $M_{t^{\prime}}$ in $I T$ are such that $\sigma$ is in both $\mathcal{D}\left(L_{t}\right)$ and $\mathcal{D}\left(M_{t^{\prime}}\right)$, then

$$
L_{t}(0)=M_{t^{\prime}}(0) \Rightarrow \delta \psi\left(\sigma ; L_{t}\right)=\delta \psi\left(\sigma ; M_{t^{\prime}}\right) .
$$

In other words, if, for each $A$ in $\mathcal{L}$, we use the symbol $A_{*}$ for an unspecified process $L_{t}$ with $A_{*}(0)=A$ and $\sigma$ in $\mathfrak{D}\left(A_{*}\right)$, then (3.24) can be written

$$
\delta \psi\left(\sigma ; A_{*}\right)=T(\sigma) \cdot A .
$$

It follows from the (local) existence theory for differential equations of the type (3.1) that, for each pair $(\sigma, A)$ with $\sigma$ in $\Sigma$ and $A$ in $\mathcal{L}$, there is at least one process $A_{*}$ such that $\sigma$ is in $D\left(A_{*}\right)$ and $A_{*}(0)=A$. Since $\delta \psi\left(\sigma, A_{*}\right)$ is, by $(3.26)$, linear in $A$, and is defined for every $A$ in $\mathcal{L}$, there is, for each $\sigma$ in $\Sigma$, a tensor $\nabla \psi(\sigma)$ in $\mathcal{L}$ (in 
fact, in ) which obeys the equation $\nabla \psi(\sigma) \cdot A=\delta \psi\left(\sigma ; A_{*}\right)$ for each $A$ in $\mathcal{E}$. For this tensor $\nabla \psi(\sigma)$ we have, again by $(3.26)$,

$$
\nabla \psi(\sigma) \cdot A=T(\sigma) \cdot A
$$

and as this holds for every $A$ in $\mathcal{L}$, there follows

$$
T(\sigma)=\nabla \psi(\sigma)
$$

which proves

Theorem 6. - Let $(\Sigma, \Pi)$ be an intrinsically equilibrated element compatible with thermodynamies. The free energy function $\psi$ for $(\Sigma, \Pi)$ bas a "gradient" $\nabla \psi: \Sigma \rightarrow \mathrm{S}$ defined by the formula

$$
\nabla \psi(\sigma) \cdot A=\delta \psi\left(\sigma ; A_{*}\right)
$$

which holds for all $A$ in $\mathcal{L}$ and $\sigma$ in $\Sigma$. In (3.29), $A_{*}$ is an arbitrary process with $A_{*}(0)=A$ and $\sigma$ in $D\left(A_{*}\right)$, and $\delta \psi(\cdot ; \cdot)$ is the function defined in (3.23). This "gradient $\nabla w$ obeys the «stress relation"

$$
T=\nabla \psi
$$

Theorems 4-6 may be regarded as broad generalization of some theorems obtained by CAPRIOLI [8] for elastic material elements. In the theory of hypo-elasticity (as described in Remark 2) Theorems 4 and 5 have the following corollary, which generalizes certain results of BERNsTEIN and ERICKSEN [9].

Remark 7. - Suppose $(\Sigma, \Pi)$ is a hypo-elastic element and is compatible with thermodynamics. Then $(\Sigma, I I)$ has precisely one free energy function $\psi$ with $\psi\left(\sigma^{\circ}\right)=0$; this function $\psi$ is lower-semicontinuous on $\Sigma \subset \mathcal{L} \oplus \mathbb{S}$ and obeys the equation

$$
\psi\left(F^{\prime}(t), S(t)\right)-\psi(F(0), S(0))=v_{r} \int_{0}^{t}|\operatorname{det} F(\tau)| S(\tau) \cdot D_{t}(\tau) d \tau
$$

Moreover, $\psi$ has a "gradient" $\nabla \psi$ as explained in Theorem 6, and the "stress relation" (3.30), which here takes the form

$$
S=\frac{1}{v_{r}|\operatorname{det} F|} \nabla \psi(\sigma)
$$

actually asserts that the stress $S$ and deformation gradient $F$ corresponding to a given state $\sigma=(F, S)$ are related by the implicit (tensorial) equation

$$
G(F, S)=0,
$$


where $G: \Sigma \rightarrow \mathcal{S}$ is determined by $\psi: \Sigma \rightarrow \mathbb{R}$ through the equation

$$
G(F, S) \stackrel{\Delta}{=} v_{r}|\operatorname{det} F| S-\nabla \psi(F, S)
$$

\section{REFERENCES}

[1] B. D. CoLEMAN - D. R. OWEN, A mathematical foundation for thermodynamics, Archive for Rational Mechanics and Analysis, 54 (1974), pp. 1-104.

[2] B. D. COLEMAN - D. R. OWEN, On thermodynamics and elastic-plastio materials, Arehive for Rational Mechanies and Analysis, 59 (1975), pp. 25-51.

[3] D. R. OWEN - W. O. WILLIAMs, On the time derivatives of equilibrated response functions, Arehive for Rational Mechanies and Analysis, 33 (1969), pp. 288-306.

[4] W. Nout, $A$ new mathematical theory of simple materials, Archive for Rational Mechanies and Analysis, 48 (1972), pp. 1-50, particularly p. 46 (\$ 20).

[5] C. TrunsdelL, Hypo-elasticity, Journal of Rational Mechanics and Analysis, 4 (1955), pp. 83-133.

[6] C. Truesdext - W. NotL, The non-linear field theories of mechanics, Handbuch der Physik, vol. III/3, ed. S. Flugge, Berlin-Heidelberg-New York: Springer-Verlag (1965), \$\$ 99-103 pp. $401-426$.

[7] B. BERNsteIn, Hypo-elasticity and elasticity, Archive for Rational Mechanics and Analysis, 6 (1960), pp. 89-104.

[8] L. CAPrioli, $8 u$ un oriterio per l'esistenza dell'energia di deformazione, Bollettino della Unione Matematica Italiana (III), 10 (1955), pp. 481-483.

[9] B. Bernstein - J. L. ERICKsen, Work functions in hypo-elasticity, Arehive for Rational Mechanics and Analysis, 5 (1958), pp. 396-409. 